\title{
ENERGETICS IN DAPHNIA PULEX POPULATIONS ${ }^{1}$
}

\author{
L. Basil Slobodkin \\ Department of Zoology, University of Michigan
}

\section{Introduction and Initial Assumption}

By combining a numerical theory of predation (Slobodkin 1957) a caloric analysis of individual Daphnia pulex (Richman 1958) and experimental data on predation in Daphnia populations it is possible to determine the energy budget and efficiency of laboratory Daphnia populations in fair detail. It will be shown that observed field efficiency data and biochemical efficiency data from the laboratory are compatible and that the entire range of reported efficiency values in the literature simply represents different ways of examining the same elementary biological process.

The experimental procedure consisted of maintaining populations of Daphnia pulex in $50 \mathrm{cc}$ of water in the laboratory at a temperature of 14$15^{\circ} \mathrm{C}$. These populations were counted every 4 days, dead animals were removed, and the total number of newborn was computed from the difference between successive censuses. A number of animals equal to some constant fraction of the number of newborn was removed every 4 days. In some cases the smallest animals present were removed first and then the stock of larger animals was tapped to make up the appropriate number.

Populations in which this procedure was followed will be referred to as "Young removal" populations. In other cases the largest animals present were removed first. These will be referred to as "Adult removal" populations. In the young removal populations removal rates were set as $50 \%$ or $90 \%$ of the newborn. In the adult removal populations removal rates were set at $25 \%, 50 \%, 75 \%$, or $90 \%$.

The number of newborn was determined as the difference between the number of living plus dead animals present at any given census, and the number of living animals present at the termination of the census and removal procedure 4 days earlier.

By assessing the number of animals missing from the population when no reproduction was occurring it was estimated that approximately $66 \%$ of the dead animals were recovered in the young removal populations and $63 \%$ were recovered in the adult removal populations. Independent experiments by Armstrong (1959) give

${ }^{1}$ This work has been supported by the Rockefeller Foundation (Ga BMR 5661), University of Michigan Faculty Research Fund (Project 993), and the National Science Foundation (G 4396). an average recovery rate of $66 \%$ per 4 days in Daphnia pulex.

For purposes of calculation the observed removal rate ( $F$ in Table $I$ ) was taken as the number of animals removed divided by the total newborn, where the number of newborn was computed as (animals alive and dead at beginning of census at time $t+1$ ) minus (animals alive at termination of censusing and removal procedure at time $t)$ and the number dead were assumed to equal the observed number dead divided by $66 \%$ in the young removal populations or by $63 \%$ in the adult removal populations.

The populations received $1,2,3,4$, or 5 rations of a water suspension of Chlamydomonas reinhardi during each 4 days. The ration was determined photometrically. By haemocytometer counts it was found that a single food ration contained $6.2 \times 10^{6}$ Chlamydomonas cells. Using Richman's figure of $1.308 \times 10^{-6}$ calories per cell, (Richman 1958) the caloric content of a single food ration was taken as 8.1 calories and will be so used throughout. The populations were kept in the dark, except during counts, so that energy fixation by uneaten Chlamydomonas was negligible.

All Daphnia were divided into the subjective size categories of large and small. The caloric content of the small animals was assumed to equal that of newborn animals as determined by Richman, while the large animals were assumed to have the caloric content of Richman's nonreproductive adult females.

The caloric content of an egg in the brood chamber of an adult female was assumed to equal $1 / 10$ th the difference between non-reproductive (or eggless) females and reproductive females (containing more than 6 eggs) as determined by Richman, since 10 eggs per female is a reasonable approximation of the number normally found in the brood pouch of a rapidly reproducing female under conditions of mass culture (Slobodkin 1954). The assumed caloric contents per individual are, therefore: for large animals .11620 calories; for small animals .012275 calories; and for each egg .017751 calories.

Details of the technique are presented in more detail in Slobodkin and Richman (1956) and Slobodkin (1954). The overall population growth patterns were essentially identical with those reported elsewhere (Slobodkin and Richman 1956) 
TABLE I. Summary of mean numerical data.

\begin{tabular}{|c|c|c|c|c|c|c|c|c|c|c|c|}
\hline \multirow[b]{2}{*}{ (1) } & \multirow[b]{2}{*}{ (2) } & \multirow[b]{2}{*}{ (3) } & \multirow[b]{2}{*}{ (4) } & \multicolumn{4}{|c|}{ Number in Residual Population } & \multicolumn{4}{|c|}{ Number in Yield } \\
\hline & & & & (5) & (6) & (7) & (8) & (9) & (10) & (11) & (12) \\
\hline Pop. & $\mathbf{F}$ & $\mathbf{N}$ & F. L. & Large & Small & Total & Eggs & Large & Small & Total & Eggs \\
\hline $\begin{array}{l}1.25 \mathrm{~A} . \\
1.50 \mathrm{~A} \\
1.75 \mathrm{~A} \\
1.90 \mathrm{~A} .\end{array}$ & $\begin{array}{l}.19096 \\
.4164 \\
.65211 \\
.83302\end{array}$ & $\begin{array}{l}82 \\
72 \\
82 \\
75\end{array}$ & $\begin{array}{l}1 \\
1 \\
1 \\
1\end{array}$ & $\begin{array}{c}33.756 \\
22.1641 \\
16.634 \\
6.800\end{array}$ & $\begin{array}{c}22.817 \\
16.8464 \\
14.793 \\
6.813\end{array}$ & $\begin{array}{l}57.024 \\
40.0105 \\
31.817 \\
13.613\end{array}$ & $\begin{array}{c}15.448 \\
14.6076 \\
10.118 \\
8.013\end{array}$ & $\begin{array}{l}1.7556 \\
3.4857 \\
4.5729 \\
3.9997\end{array}$ & $\begin{array}{r}.2072 \\
.2777 \\
.8902 \\
1.9599\end{array}$ & $\begin{array}{l}1.9628 \\
3.7634 \\
5.4631 \\
5.9596\end{array}$ & $\begin{array}{l}2.0350 \\
6.4016 \\
8.5357 \\
5.5590\end{array}$ \\
\hline $\begin{array}{l}3.25 \mathrm{~A} . \\
3.50 \mathrm{~A} . \\
3.75 \mathrm{~A} \text {. } \\
3.90 \mathrm{~A} .\end{array}$ & $\begin{array}{l}.20027 \\
.40884 \\
.69726 \\
.90148\end{array}$ & $\begin{array}{l}82 \\
82 \\
82 \\
68\end{array}$ & $\begin{array}{l}3 \\
3 \\
3 \\
3\end{array}$ & $\begin{array}{r}96.794 \\
74.390 \\
32.414 \\
4.941\end{array}$ & $\begin{array}{r}65.049 \\
52.403 \\
24.146 \\
7.074\end{array}$ & & $\begin{array}{r}34.953 \\
35.695 \\
13.793 \\
7.631\end{array}$ & & $\begin{array}{r}.3048 \\
.4999 \\
.6584 \\
2.6764\end{array}$ & $\begin{array}{l}4.9264 \\
9.5850 \\
9.6337 \\
7.4407\end{array}$ & $\begin{array}{r}6.3405 \\
15.1700 \\
17.8530 \\
8.5725\end{array}$ \\
\hline $\begin{array}{l}5.25 \mathrm{~A} . \\
5.50 \mathrm{~A} \\
5.75 \mathrm{~A} \\
5.90 \mathrm{~A}\end{array}$ & $\begin{array}{l}.21909 \\
.43631 \\
.68361 \\
.85104\end{array}$ & $\begin{array}{l}82 \\
82 \\
82 \\
64\end{array}$ & $\begin{array}{l}5 \\
5 \\
5 \\
5\end{array}$ & $\begin{array}{r}164.750 \\
63.354 \\
15.057 \\
8.716\end{array}$ & $\begin{array}{r}115.344 \\
49.450 \\
12.889 \\
10.296\end{array}$ & $\begin{array}{r}284.056 \\
112.950 \\
28.141 \\
19.058\end{array}$ & $\begin{array}{r}66.609 \\
36.233 \\
9.916 \\
12.446\end{array}$ & & $\begin{array}{r}.3703 \\
.9146 \\
1.8658 \\
3.3593\end{array}$ & $\begin{array}{r}8.9865 \\
9.6947 \\
7.6094 \\
10.0126\end{array}$ & $\begin{array}{l}13.9845 \\
18.3159 \\
12.5970 \\
14.5253\end{array}$ \\
\hline $\begin{array}{c}1.50 \mathrm{Y} . \\
2.50 \mathrm{Y} \\
2^{\prime} .50 \mathrm{Y} \\
4.50 \mathrm{Y} \\
5.50 \mathrm{Y}\end{array}$ & $\begin{array}{l}.4219 \\
.4385 \\
.4344 \\
.4351 \\
.4426\end{array}$ & $\begin{array}{l}82 \\
82 \\
81 \\
81 \\
82\end{array}$ & $\begin{array}{l}1 \\
2 \\
2 \\
4 \\
5\end{array}$ & $\begin{array}{r}30.422 \\
60.823 \\
56.272 \\
120.236 \\
153.899\end{array}$ & $\begin{array}{l}14.657 \\
35.665 \\
34.150 \\
73.412 \\
89.036\end{array}$ & $\begin{array}{r}45 . \\
97 . \\
90 . \\
195 . \\
243 .\end{array}$ & $\begin{array}{l}23.766 \\
40.239 \\
55.768 \\
68.709 \\
86.699\end{array}$ & $\begin{array}{r}.1705 \\
.0617 \\
.2500 \\
.2125 \\
1.6460\end{array}$ & $\begin{array}{r}5.7926 \\
11.1974 \\
11.5375 \\
21.2375 \\
22.0852\end{array}$ & $\begin{array}{r}5.9631 \\
11.2591 \\
11.7875 \\
21.4500 \\
23.7312\end{array}$ & $\begin{array}{c}.0363 \\
0 \\
.0250 \\
.5250 \\
2.4384\end{array}$ \\
\hline $\begin{array}{r}1.90 \mathrm{Y} \ldots \ldots \ldots \\
2.90 \mathrm{Y} \ldots \ldots \ldots \ldots \\
2^{\prime} .90 \mathrm{Y} \ldots \ldots \ldots \\
4.90 \mathrm{Y} \ldots \ldots \ldots \\
5.90 \mathrm{Y} \ldots \ldots \ldots\end{array}$ & $\begin{array}{l}.8424 \\
.8535 \\
.8568 \\
.8532 \\
.8678\end{array}$ & $\begin{array}{l}74 \\
74 \\
68 \\
68 \\
66\end{array}$ & $\begin{array}{l}1 \\
2 \\
2 \\
4 \\
5\end{array}$ & $\begin{array}{l}11.278 \\
13.914 \\
28.405 \\
52.012 \\
21.572\end{array}$ & $\begin{array}{r}3.742 \\
4.150 \\
9.151 \\
24.059 \\
10.135\end{array}$ & $\begin{array}{l}15.060 \\
18.146 \\
37.617 \\
76.220 \\
31.737\end{array}$ & $\begin{array}{r}26.930 \\
29.878 \\
50.715 \\
115.277 \\
49.672\end{array}$ & $\begin{array}{r}.8646 \\
1.3419 \\
.7423 \\
1.4177 \\
.6029\end{array}$ & $\begin{array}{l}17.6215 \\
20.0547 \\
34.2726 \\
69.8507 \\
36.1666\end{array}$ & $\begin{array}{l}18.4861 \\
21.3966 \\
35.0149 \\
71.2684 \\
35.7695\end{array}$ & $\begin{array}{r}.5670 \\
.6835 \\
.1211 \\
.2536 \\
1.1541\end{array}$ \\
\hline $\begin{array}{l}0.1 \ldots \\
0.2 \ldots \\
0.2^{\prime} \\
0.4 \ldots \\
0.5 \ldots\end{array}$ & $\begin{array}{l}0 \\
0 \\
0 \\
0 \\
0\end{array}$ & $\begin{array}{l}82 \\
70 \\
81 \\
81 \\
82\end{array}$ & $\begin{array}{l}1 \\
2 \\
2 \\
4 \\
5\end{array}$ & \begin{tabular}{r|}
36.642 \\
66.981 \\
72.774 \\
146.182 \\
176.216
\end{tabular} & $\begin{array}{r}29.950 \\
54.499 \\
53.542 \\
105.481 \\
116.731\end{array}$ & $\begin{array}{r}66.641 \\
122.665 \\
127.316 \\
254.502 \\
294.715\end{array}$ & $\begin{array}{l}19.257 \\
37.076 \\
33.075 \\
55.859 \\
93.896\end{array}$ & $\begin{array}{l}\mathbf{0} \\
\mathbf{0} \\
\mathbf{0} \\
0\end{array}$ & $\begin{array}{l}0 \\
0 \\
0 \\
0 \\
0\end{array}$ & $\begin{array}{l}- \\
- \\
-\end{array}$ & $\begin{array}{l}0 \\
0 \\
0 \\
0\end{array}$ \\
\hline
\end{tabular}

The entries in the columns are:

Column (2)-F, the removal rate estimated from births, deaths and censuses, corrected for decomposition of the dead.

Column (3)-Number of censuses.

Column (4)-Food level, number of feedings in a four day period.

Column ( 5)-Mean number of large animals in the residual population.

Columo 6)-Mean number of small animals in the residual population. This is the sum of columns (5), (6) and the mean number of males.

Column (8)-Mean number of eggs in the reproductive females of the residual population.

Column (9)-Mean number of large animals in the yield per four day interval.

Column (9)-Mean number of large animals in the yield per four day interval.

Column (10)-Mean number of small animals in the yield per four day interval.

Column (12)-Mean number of egss contained in the reproductive females in the yield per four day interval.

and will not be reported in detail here. The discussion will center around the mean values of population size and yield from 22 experimental populations and 5 controls. The number of consecutive censuses available for each population is indicated as $N$ in Table I. Each population from which animals were removed is designated by an initial integer, indicating the food level followed by a 2 digit number representing the $a$ priori rate of removal and either an $A$ indicating that adults were preferentially removed or a $Y$ indicating that small animals were selected. For example, population $3.75 \mathrm{~A}$ received 24.3 calories of food every 4 days and was subjected to removal of the largest possible adult animals at the rate of approximately $75 \%$ of the number of newborn.

The control populations are indicated by zero followed by the food level. For example, 0.2 is the control population which received 2 food rations every 4 days. Note that the pairs $2.50 Y$ and $2^{\prime} .50 \mathrm{Y}, 2.90 \mathrm{Y}$ and $2^{\prime} .90 \mathrm{Y}$, and 0.2 and $0.2^{\prime}$ were treated identically.

By residual population (or standing crop) is meant the animals that remained in the population after the completion of the censusing and removal process. The animals removed are referred to as the yield. Note that the number of eggs in the brood chambers were counted and summed for each population. In Tables I and II the numerical and caloric means for residual population and yield are indicated for each of the 27 populations.

The males present were added separately to the total residual population. In general, males were not removed as yield but some may be included with the small yield animals. 
TABLE II. Summary of caloric contents, energy inputs, efficiencies and replacement rates. The entries in the columns are:

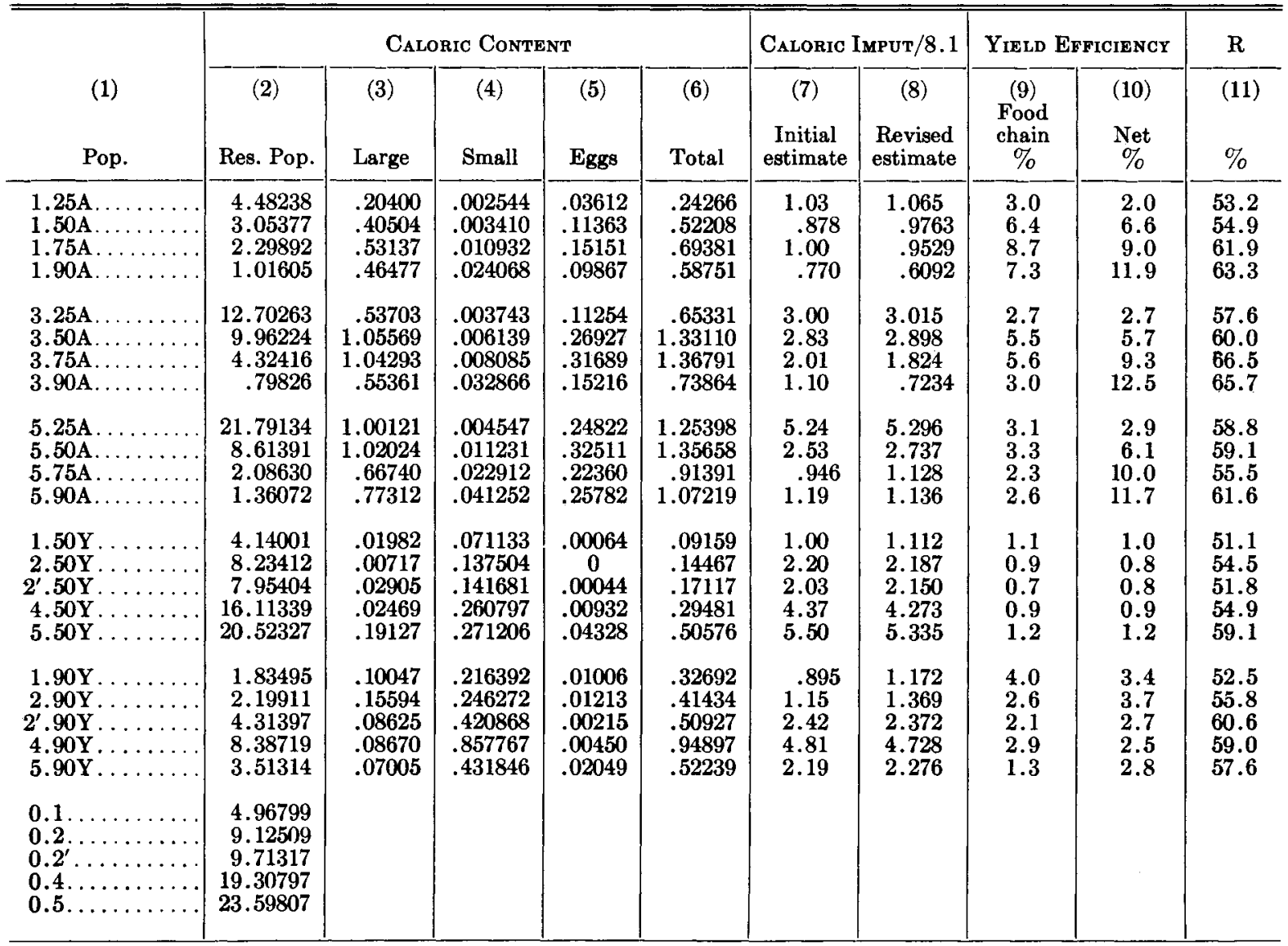

Column ( 1)-Code name of the populations.

Column ( 2)-Caloric content of the mean residual population.

Column (3)-Caloric content of the adult animals in the yield per four days

Column (4)-Caloric content of the small animals in the yield per four days.

Column ( 5)-Caloric content of the egge in the yield per four days.

Column ( 6)-Total caloric yield per four days, the sum of column s (5), (4) and (3).

Column ( 7)-Caloric input divided by 8.1 as estimated from equation (2), a revised estimate of the food level

Column ( 8)-Caioric input divided by 8.1 as estimated from equation (3) for each population.

Column (9)-Food chain yield efficiency. Each entry is equal to the corresponding entry in column (6) divided by 8.1 times the uncorrected food level

Column (10)-Net yield effieiency. Esch entry is equal to the corresponding entry in column (6) divided by 8.1 times the entry in column (8).

Column (11) - The percent of the protoplasm in the residual populations which is repiaced during a four day period. Replacement rate is calculated from equation (7).

\section{ANALysis of the Data}

\section{Approximate energy consumed}

Let $P_{0}$ represent the size of a control population, $P_{F}$ the size of a population from which animals are removed at the rate $F$. Assume population size to be linearly dependent on food supply at all food levels and removal rates, and also assume that the energetic cost to the population of each animal removed as yield is essentially independent of the age and size of the animals removed. The assumption of linearity is justified by previous studies of Daphnia populations, both in the laboratory (Slobodkin 1954, 1957) and in the field (Borecky 1956) and will be discussed in detail below.

Let :

$$
\frac{P_{F}}{P_{O}}=\varphi(F)
$$

We require that when $F=0, P_{F}=P_{0}$ and $\varphi(F)=1$; when $F=1, P_{F}=0$ and $\varphi(F)=0$. At intermediate values of $F$, the reproductive rate of $P_{F}$ tends to compensate for removal of animals so that $P_{F}$ is a non-linear function of $F$, with the absolute value of $\frac{-\mathrm{dP}_{F}}{\mathrm{dF}}$ increasing as $F$ approaches 1.

A suitable value of $\varphi(F)$, that has the appropriate limits and is in general agreement with the known compensating mechanisms of Daphnia populations is :

$$
\frac{P_{F}}{P_{O}}=1-\frac{F}{2-F}
$$


This equation has been derived elsewhere from empirical population variability data (Slobodkin 1957 ) and is in reasonable agreement with the observed values of $P_{F}$ for the young removal populations (Figure 1) but not for the adult removal populations (Figure 2). Note that for the young removal populations the assumption of linearity with food supply is approximately valid for both population size (Figure 1) and yield expressed either as total number of animals (Figure 3 ) or animals plus eggs (Figure 5 ).

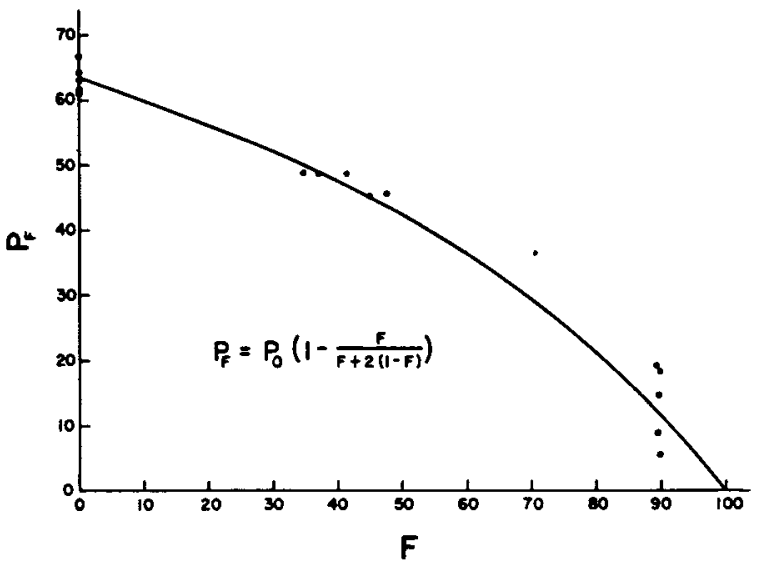

FIG. 1. Young removal populations. Residual population size per unit food supply plotted against $F$. The line is drawn from equation (1).

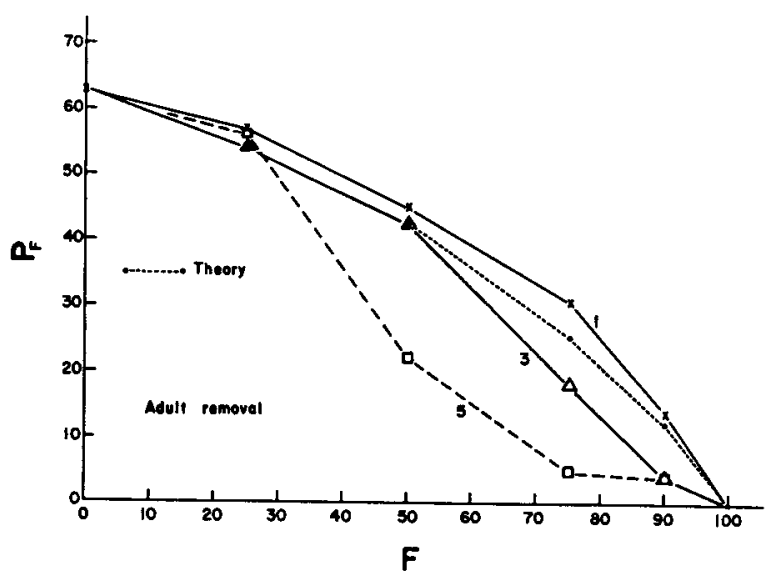

Fig. 2. Adult removal populations. Residual population size per unit food supply plotted against $F$. The dotted line is drawn from equation (1). The first food level is drawn as $-\mathrm{x}-$, the third as $-\Delta-$ and the fifth as $-\square-\square$.

Yield and population size are not linearly related to food supply in the adult removal populations. Since microscopic examination indicated that Chlamydomonas cells were consistently present in the used medium from populations $3.75 \mathrm{~A}$, $3.90 \mathrm{~A}, 5.50 \mathrm{~A}, 5.75 \mathrm{~A}$, and $5.90 \mathrm{~A}$ but not in the used medium from the other populations, it is

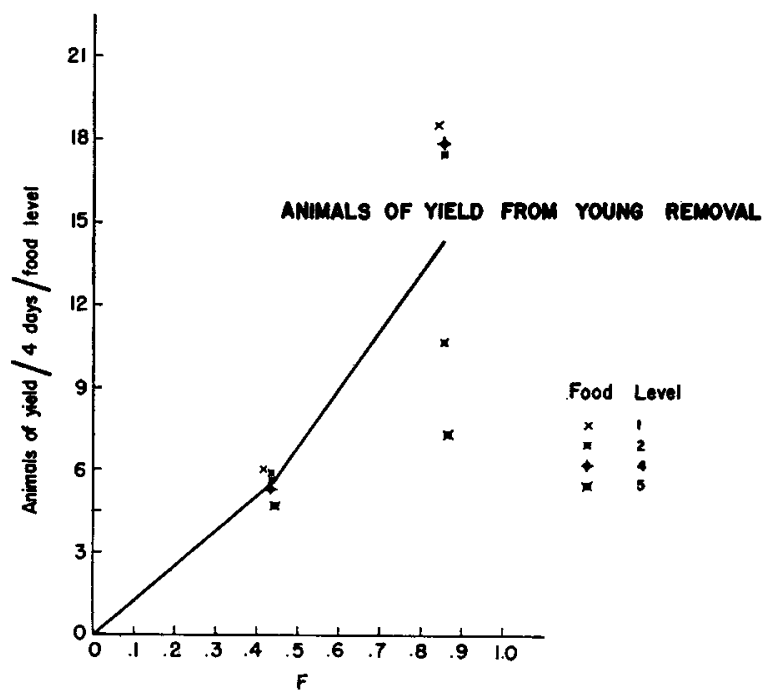

FIG. 3. Young removal populations. Mean yield in animals per census per unit food supply plotted against $F$. With the exception of populations $2.90 \mathrm{Y}$ and $5.90 \mathrm{Y}$, yield per unit food supply seems essentially constant for each value of $F$.

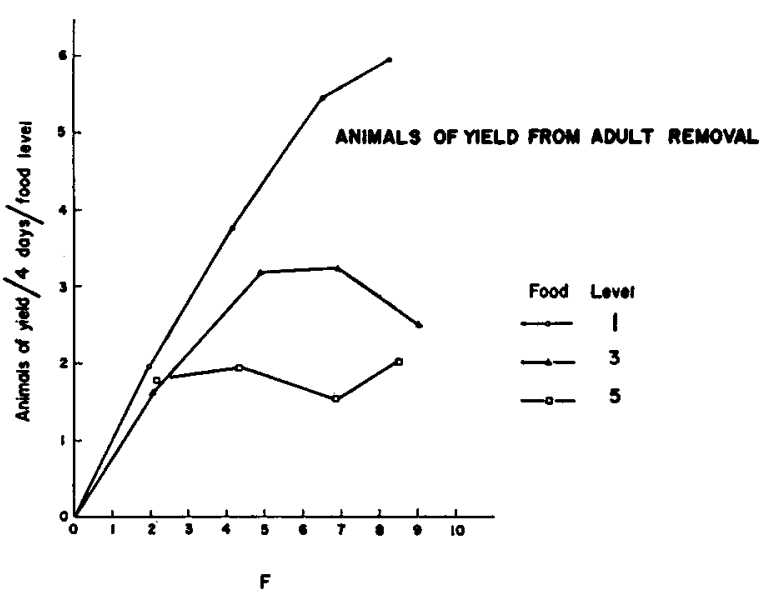

FIG. 4. Adult removal populations. Mean yield in animals per census per unit food supply plotted against $F$. Yield per unit food supply decreases with food level execept at the lowest values of $F$. For any given value of $F$ the number of animals in the yield is lower in the adult removal populations than in the young removal populations. The lowest food level populations are indicated by circles, the third food level by triangles and the fifth food level by squares.

clear that in at least some of the populations the food supplied was not equivalent to the food consumed. There is ample experimental evidence that population size in Daphnia is linearly related to food consumption for the case in which all the food supplied is actually consumed (Slobodkin 1954). We should therefore be able to use the population size to determine the relative food consumption by considering that any deviation from a linear relation between population size and food 


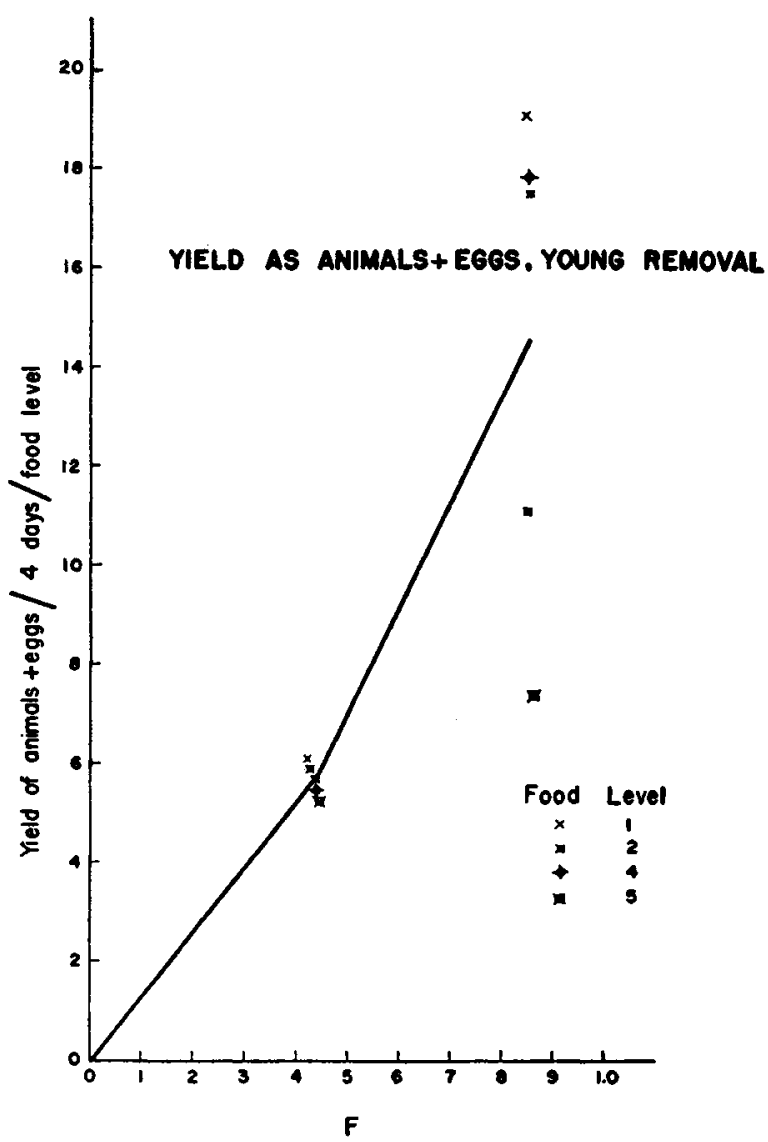

FIG. 5. Young removal populations. Mean yield in animals and eggs per census per unit food supply. The pattern is essentially the same as in Fig. 3.

supply is proportional to the difference between the food supplied and the food actually consumed. It will therefore be assumed that all of the populations are actually following equation (1) but that the food supplied to the population is not necessarily identical with the food ingested by the populations. Analysis of an experimental test of this assumption is now in progress. We will assume that the food consumed by a population is measured by its deviation from the calculated value as determined from $F$ (Table I) and equation (1) according to the following equation:

$$
\frac{P_{F}}{\text { calculated } P_{F}}=\text { Food consumed }
$$

The calculated food consumed for each population is listed as Column 8 of Table II and is equal to $1 / 8.1$ times the calories consumed, to a first approximation.

If we now replot yield per calculated food consumption level it is found that it is essentially dependent only on $F$ (Figures 7 and 8). Furthermore, if we compute production efficiency (see below) using the food supply as our energy input we find negative efficiency values; while production efficiency values derived from the calculated food consumption are all positive and in agreement with other estimates of biological efficiency. That is, failure to use equation (2) leads to nonsense conclusions, while the conclusions derived from equation (2) are simple and in remarkably close agreement with other, independent, data. We therefore consider that the food input derived from equation (2) is a closer approximation to the actual food ingested than is the raw food level data.

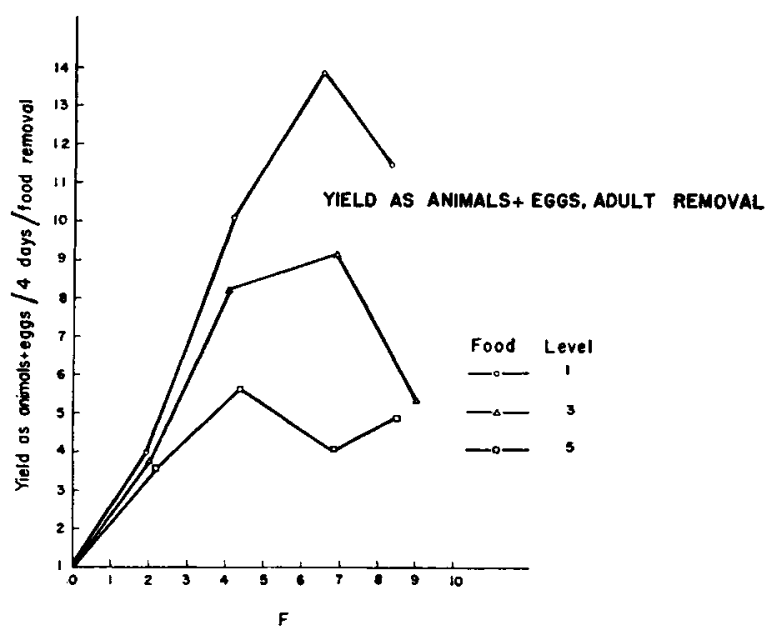

FIG. 6. Adult removal populations. Mean yield in animals and eggs per census per unit food supply. The pattern is essentially the same as in Fig. 4, except that addition of eggs to the yield makes the low food level, adult removal yield comparable in numbers with the young removal yields.

\section{Efficiency}

Having values for the caloric content of the yield and the caloric content of the food supplied, the simple quotient (calories of yield divided by 8.1 times food level) is equal to the efficiency with which the Daphnia transform the Chlamydomonas into food for a hypothetical predator. This quantity is listed as Column 9 of Table II. This is the "food-chain"2 efficiency and is plotted as a function of $F$ in Figures 9 and 10.

Food-chain efficiency increases with $F$ for the young removal populations but has a maximum at intermediate values of $F$ in the adult removal populations. This is simply due to the inability of the greatly reduced residual adult populations to consume all of the food provided. Removal of young does not as severely lower food consuming capacity. The peak value of food-chain efficiency diminishes with increasing food concentration as does the maximally efficient value of $F$. It might

"This term is borrowed, with permission, from a book manuscript by L. C. Cole. 


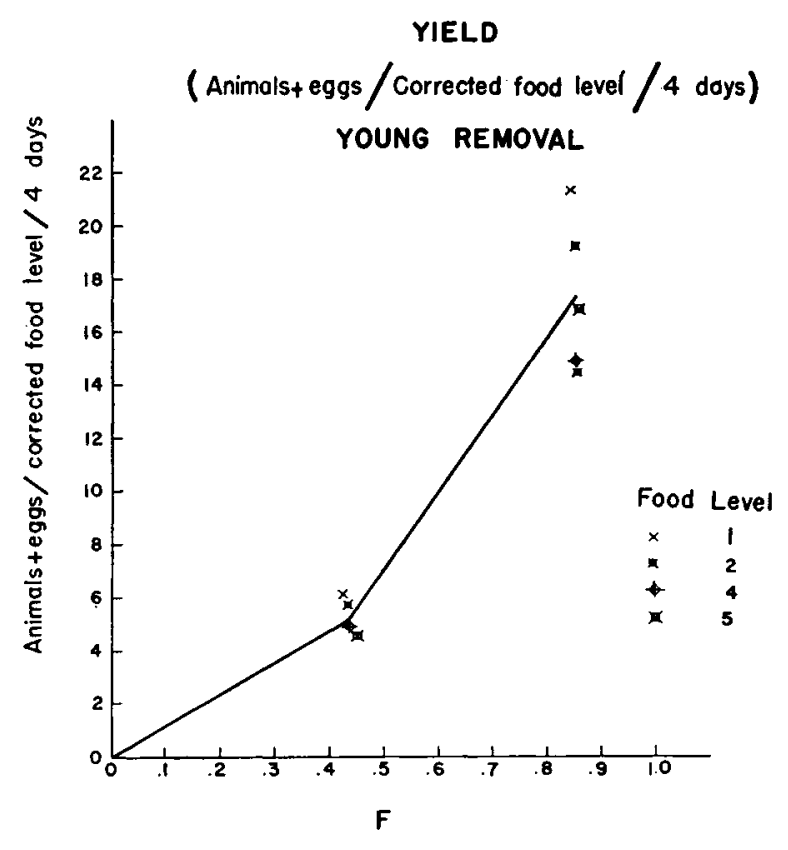

FIG. 7. Young removal populations. Mean yield in animals and eggs per census per unit food consumed as determined from equation (2). Scatter is reduced at high levels of $F$.

YIELD

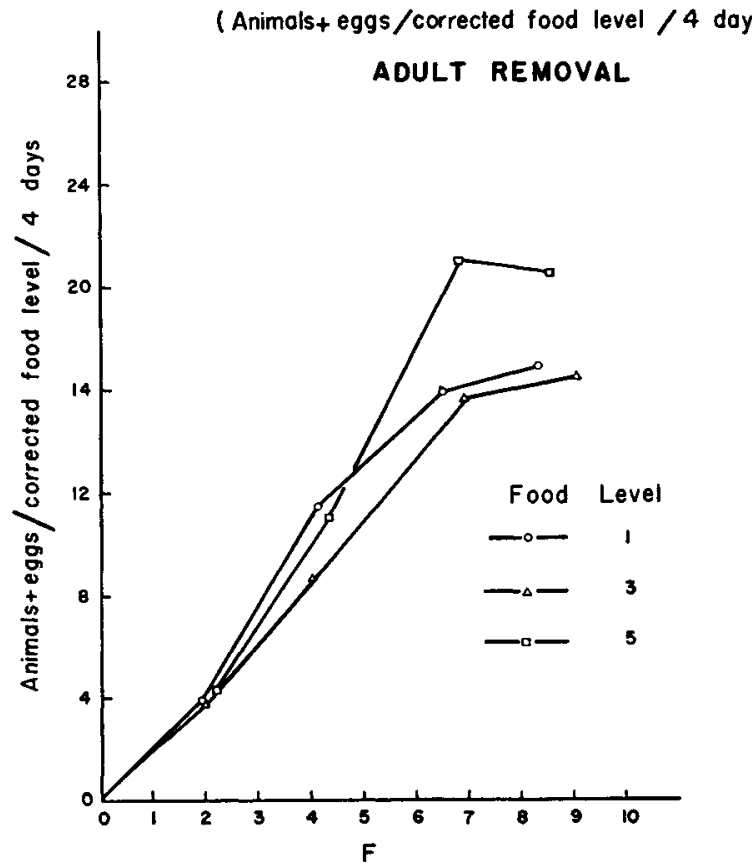

FIG. 8. Adult removal populations. Mean yield in animals and eggs per census per unit food consumed as determined from equation (2). The disparity between food levels has disappeared.

be expected, with more dilute algal concentrations, that peak values of $10-12 \%$ might be achieved. These values are in general agreement with those reported in nature. (Lindeman 1942,

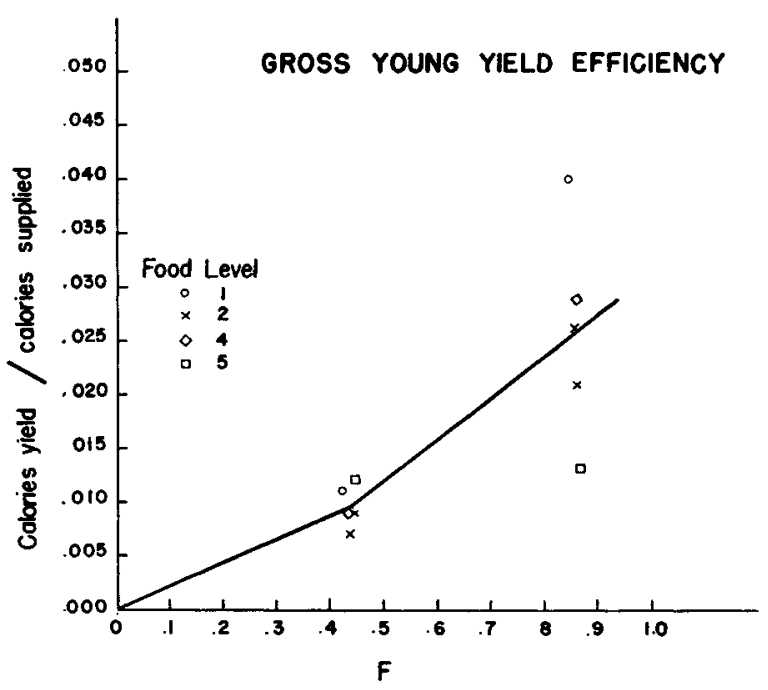

FIG. 9. Young removal populations. Food chain efficiency plotted against $F$. Food chain efficiency is defined as the total yield in calories divided by the total calories of food supplied to the population.

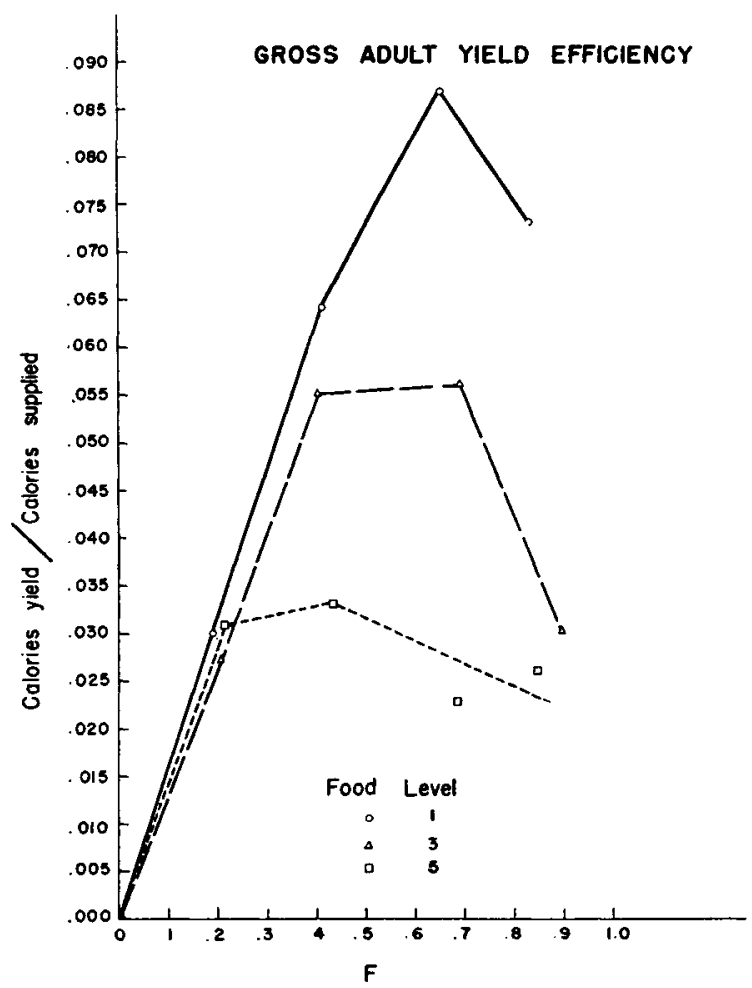

FIG. 10. Adult removal populations. Food chain efficiency plotted against $F$. At all food levels food chain efficiency has a maximum at some intermediate value of $F$ and the maximally efficient value of $F$ decreases with food level as does the maximum food chain efficiency attained.

Wright 1958, Juday 1940) It is definitely clear that, if any predator feeds preferentially on young Daphnia, the Daphnia population will not be more than approximately $6 \%$ efficient at producing 
yield for that predator. In addition to the foodchain efficiency it is of interest to determine the net efficiency, that is, the ratio of calories of yield to calories of food actually consumed. The specific efficiencies of reproduction, growth, and the caloric cost of population maintenance (in calories/4 days/calorie) are also significant.

These will be derived from an analysis of caloric data as follows: The energy budget of a Daphnia population can be written as:

calories of food consumed per 4 days $=[$ (cost, in calories per 4 days, of maintaining each calorie of standing crop) $\times$ (the caloric content of the standing crop $)]+[($ cost per calorie of producing a calorie of yield) $\times$ (caloric content of the yield per 4 days.)]

Each of the 22 populations subject to predation must follow equation (3) and for each population we know the caloric content of the steady state population and of the adult, young, and egg yield. We also have an estimate of the calories of food consumed per 4 days. It is therefore possible to solve by least squares for the caloric cost of maintenance and the efficiency of production of adult, young, and egg yield. Details of the solution are presented in the Appendix and Table III. A second approximation to the caloric input to each population is obtained by substituting the best least squares values for the maintenance costs and efficiencies back into equation (3) with the known steady state caloric contents and yields and solving for input. This revised estimate of the calories consumed for each population is listed as Column 8 of Table II.

Using the calculated cost and efficiencies, equation (3) can be rewritten for each population as caloric input per four days =

$$
\begin{gathered}
\frac{\text { caloric content of standing crop }}{.5938 \text { cal. days per cal. }}+ \\
\left(\frac{\text { calories of adult yield per } 4 \text { days }}{.4830}+\right. \\
\frac{\text { calories of egg yield per } 4 \text { days }}{.06204}+ \\
\left.\frac{\text { calories of young yield per } 4 \text { days }}{.03586}\right)
\end{gathered}
$$

Net efficiency can now be defined as (total calories of yield per 4 days divided by the revised estimate of energy input per 4 days). For the adult removal populations this is a linear function of $F$, while for the young removal populations there is some indication of higher net efficiency at higher values of $F$ (Figure 11). The lack of complete linearity in the young removal populations is probably due to the fact that a higher

\begin{tabular}{|c|c|c|c|c|c|}
\hline & (1) & (2) & (3) & (4) & \\
\hline & 1809.49308530 & 65.75403750 & 16.79684106 & 24.32872472 & (1) \\
\hline \multirow[t]{3}{*}{$A=$} & 65.75403750 & 6.68031307 & 1.88140823 & .38309611 & (2) \\
\hline & 16.79684106 & 1.88140823 & .54208247 & .06774019 & (3) \\
\hline & 24.32872472 & .38309611 & .06774019 & 1.39675661 & (4) \\
\hline \multirow[t]{4}{*}{$A-1=$} & .0013496 & -.0509634 & .1370824 & -.0161774 & (5) \\
\hline & -.0509634 & 9.347013 & -30.839049 & -.1803367 & (6) \\
\hline & .1370824 & -30.839049 & 104.505111 & 1.0023809 & (7) \\
\hline & -.0161774 & -.1803367 & 1.0023809 & .998571 & (8) \\
\hline$g_{i}=$ & 510. 16442814 & 20.44016716 & 5. 28479188 & 10.098613286 & (8) \\
\hline \multirow{4}{*}{$\frac{8.1}{x_{i}}=$} & .207900 & .25569 & 1.98967 & 3.44231 & $(10)$ \\
\hline & & & & & \\
\hline & $y=.5938$ & $x_{1}=.4828$ & $x_{2}=.06204$ & $x_{3}=.03586$ & (11) \\
\hline & $y^{\prime}=.5938$ & $x_{1}^{\prime}=.4830$ & $x_{2}^{\prime}=.06204$ & $\mathrm{x}_{3}{ }^{\prime}=.03586$ & (12) \\
\hline
\end{tabular}
proportion of adult animals are removed from
Table III. Matrix solution for the values of $y$ and the $x_{i}$ in equation (4).

The first four rows are the coefficients of equation (4), e.g., the entry in the second column, third row is the sum of the products $b_{1} b_{2}$ for all twenty-two experimental populations where $b_{1}$ is the caloric content of the adult yield and $b_{2}$ is the caloric content of the egg yield, as indicated in Columns (3) and (4) of Table II The diagonal elements are the sums of the squares of the caloric data for standing crop (a11), adult yields, (a22), egg yield ( $\mathbf{a}_{33}$ ) etc.

Rows (5) through (8) are the inverse of the matrix consisting of the first fout rows.

Row (9) contains the sums of the products of a or the (bi) multiplied by food level as estimated from equation (2) for each population, e.g., the entry in the third column of row $(9)$ is the sum of the products of columns $(5)$ and $(7)$ in Table II Row (10) is the vector resulting from the matrix multiplication of row $(9)$ and the inverse matrix (A-1) rows (5) through (8).

Row (11) contains the initial estimates of $y$ and the $x_{i}$ values in equations (3) and (4) of the text.

Row (12) contains the values of $y^{\prime}$ and $x^{\prime}$ obtained by substituting the values of $y$ and $x_{i}$ into equation (3), solving for I and re-computing row (9). The values of used for getting $y^{\prime}$ and $x_{i}^{\prime}$ are listed as Column (g) of Table II.

these populations at high levels of $F$. Note that the linearity of net efficiency with $F$ depends on the assumed constancy of the efficiencies and costs. This assumption is supported by other evidence indicating that all quantities in unexploited Daphnia population are linearly proportional to food supply (see especially Slobodkin 1954). If this assumption is not valid there will probably be a slight upward convexity to the relation at high levels of $F$ since our major revision of input calorie estimates occurred at these levels. The values of production efficiency derived from the matrix indicate that if all available energy was utilized for the production of young, new calories of young protoplasm would be produced with an efficiency of $3.6 \%$, the corresponding effciency for egg production is slightly higher, that is $6.2 \%$. The difference is presumably due to the cost of differentiating the egg protoplasm into a new animal. The efficiency of producing adult protoplasm, in the sense of adding new protoplasm to an extant animal, is $48 \%$, which seems remarkably high at first glance. In particular, the work of Hutchens (1951) would indicate a steady state efficiency of not more than $25-30 \%$ for glucose metabolism.

Even more elementary considerations would indicate that an efficiency of more than $30 \%$ is 


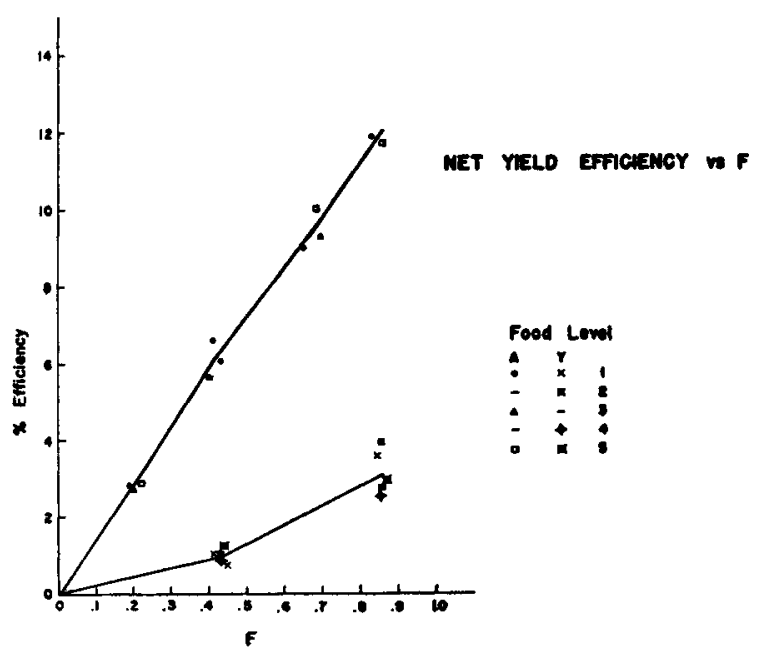

FiG. 11. Net efficiency, all populations. Calories of yield per census divided by the final estimate of calories consumed per census interval (i.e. $8.1 \times$ column 8 , Table II) plotted against $F$. The relation is essentially linear for the adult removal populations and concave upward for the young removal populations.

impossible in a steady state biological system. Consider that at least 150 chemical steps are involved in the complete degradation of any food molecule and that there must be a sufficient number of exergonic steps in the sequence to guarantee unidirectionality of metabolism. The energy burned at each step is unavailable for growth. To leave sufficient residual energy to permit an efficiency of much more than $25 \%$ requires an absurdly high mean efficiency per step. The precise value is given by: $n \log e=\log E$ where $n$ is the number of steps, $e$ the mean efficiency per step, and $E$ the overall efficiency of growth. The apparent contradiction is resolved when it is considered that the $48 \%$ efficiency value is not a steady state value, since it does not account for maintenance. The effect of removing an animal from a population is twofold. Most obvious is the actual cost of producing the animal. There is also a saving of the energy that would have been required to maintain that animal in the population had it not been removed. The production cost, as determined by the matrix, cannot distinguish between these two effects. Since the cost for maintaining a calorie of protoplasm for 4 days is known (i.e., .5938 cal.) the true efficiency of growth including maintenance costs can be determined."

On the assumption that maintenance cost per calorie is constant, the maintenance cost per 4 days for a single adult Daphnia is $\frac{0.1162}{.5938}$ or 1957 calories. The net cost of removing the same animal from the population is $\frac{0.1162}{.4830}$ or .2406 calories, so that the total cost, for each adult is .4363 calories, and, since the caloric content of each adult is .11620 calories, the true efficiency of growth, taking maintenance into account is $26.63 \%$, which is in agreement with the biochemical requirements.

Corresponding arguments apply to the efficiency of production of eggs and young. Several alternative assumptions are available. We almost certainly must assume that the maintenance cost of eggs is paid by the adult that produced those eggs in its ovary and this is conceivably also valid for young, but because of variations from population to population in the mean number of eggs per adult, we will simply add the maintenance cost to the young and eggs themselves. Owing to the small size of the young and eggs the quantitative effect is negligible, altering cost per animal from .3423 to .3630 calories in the young and correspondingly shifting efficiency from a production efficiency of $3.59 \%$ to a true efficiency of $3.38 \%$. Egg costs per egg shift from .2861 to .3160 and the true efficiency of egg growth is now $5.62 \%$ rather than the production efficiency of $6.20 \%$.

By using the calculated mean efficiency and maintenance cost values (Appendix, A) and the observed census and yield data from each population it is possible to calculate the rate at which calories are replaced in the residual populations. The details of this calculation are in the Appendix (B). The calculated replacement rates for each population are listed as $R$ in Column 11, Table II. Replacement rate $(R)$ is essentially constant and equal to 57.95\%. Richman (1958) measured the weight lost by starved Daphnia and found approximately $56 \%$ weight loss in 4 days. To maintain a steady state, replacement would have to be $56 \%$ at least from his data. The agreement with the present estimate is so good as to be very surprising. It should be possible to check the theory by calculating the caloric content of the control populations from maintenance cost $(y)$ as estimated from the energy budget, since, in the absence of predation, equation $(3)$ reduces to

calories of food consumed per 4 days $=$ caloric content of the standing crop .5938 cal. days per cal.

or

$.5938 \times 8.1=4.69$ cal. of standing crop per unit food in the control populations. The observed mean caloric content of the control populations is 4.77 . 


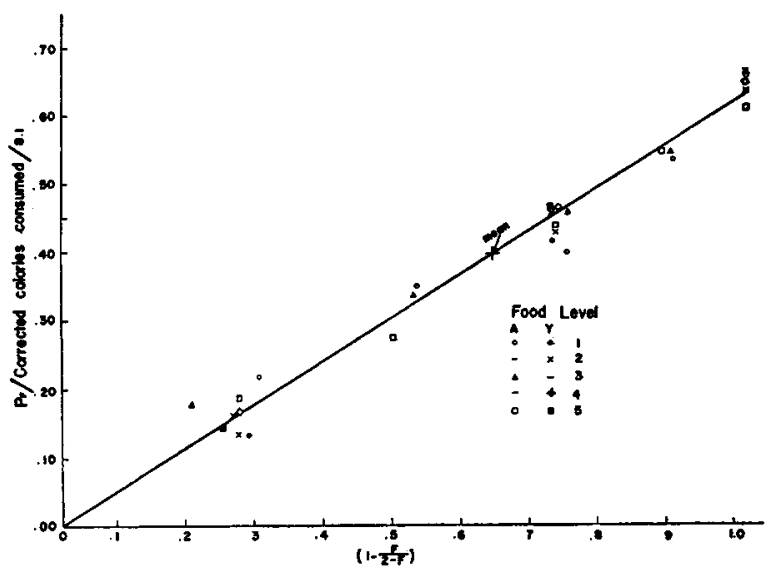

FIG. 12. Residual population size divided by the final estimate of food consumed plotted against $\left(1-\frac{F}{2-F}\right)$.

The line has been drawn through the origin and the mean of the points. This is equivalent to Figs. 1 and 2 after re-evaluation of the food consumption for each population.

Note that the control populations influenced the result of the matrix analysis only in the original calculation of energy input from equations (1) and (2).

A similar result is attained if we substitute the mean replacement rate, or Richman's weight loss data from an individual Daphnia for the maintenance cost. That is, we have found 3 numbers that are identical with each other. These are:

1. The percentage weight loss in 4 days by a starved adult Daphnia (56\%).

2. The inverse of maintenance cost per 4 days per calorie estimated as a weighting constant in an energy budget $(59 \%)$. (See Appendix A.)

3. The replacement rate per 4 days per calorie estimated on the assumption that all the energy which is not utilized in providing yield is used in replacement reproduction and replacement growth $(58 \%)$. (See Appendix B.)

As a matter of convenience we have referred to the inverse of maintenance cost and the replacement rate but both concepts are defined in the identical units of calories per calorie day. The clear implication is that the same thing has been measured in three different ways. This conclusion is equivalent to stating that the process of maintaining a steady state Daphnia population, regardless of its size (since we have the data from the limiting case of a single animal) consists exclusively of growing or reproducing sufficient new protoplasm to replace the loss in death plus catabolism. This very simple conclusion may be amplified slightly.
It is possible, in principle, to consider the process of maintenance in a biological system in two different ways. We could imagine that there is a certain cost involved in maintaining a mass of protoplasm and that this cost is not related to the growth or reproductive characteristics of the system but in some way is a third property of the system. The other alternative is that growth and reproduction are identical with the processes involved in population increase, size increase of individual animals or production of yield for a predator and are the only processes that are significant in maintenance of a biological steady state.

If the first alternative were correct we would have expected the maintenance cost per 4 days, as determined from the energy budget, to differ from the replacement rate per 4 days as determined from growth and reproductive efficiency data and the census data for each population.

As a matter of fact, maintenance cost and replacement rate are identical. We must therefore conclude that growth and reproduction are identical with steady state population maintenance in Daphnia. An equivalent statement would be that the only direct source of usable energy for metabolic work in a Daphnia population is Daphnia protoplasm and that this protoplasm is produced with a fixed efficiency regardless of its eventual fate.

\section{Possible Second Order Corrections}

The surprisingly close agreement between replacement rate per 4 days as calculated and the observed weight loss in starved Daphnia and between observed and calculated control population size indicate that the efficiency values are fairly accurate. It is of particular interest to note that the widely different values that "efficiency" may take in the same system depend on precisely how efficiency is defined.

While the possible sources of error are probably obvious it seems useful to list them here. There may be quantitative inaccuracies in:

1) The original estimate of decomposition rate of dead animals.

2) The assumed caloric contents of the animals and algae.

3) The general assumption of universal linearity.

4) The assumption that both young and adult removal situations ideally correspond to equation (1).

Of these the most important is probably the fourth. Apparently the general agreement of equation (1) with both young and adult removal data is primarily a very fortunate accident. The 
cost of young, adults, and eggs are sufficiently close to equality, and the numbers in the yield happen to balance out so that the effect on residual population size is virtually independent of the age and size of the animals removed. By any set of reasonable assumptions it would also be the case that removal of an adult animal from a population reduces the filtering rate of that population considerably more than removal of a young animal or an egg. This is not significant unless the filtering rate is reduced below some critical value. At this point food is left over in the medium. This point will be analyzed in greater detail in connection with another set of experiments. In addition, the efficiency of assimilation tends to vary inversely with food concentration. (Richman 1958) so that even if all the food is eaten there will be effectively lower food absorption at high food levels. These effects are simultaneously compensated for in part by our assumption of the general validity of equation (1). We must concede that equation (1) is not generally applicable to other species and that it should be replaced by a more general statement.

Equation (3) is a general statement in caloric terms. This can fairly readily be translated into numerical terms. A rather complex replacement for equation (1) could then be constructed following the same type of argument as that of Beverton and Holt (1957). It will not be attempted here. Equation (1) is approximately valid whenever the values of efficiency in equation (3) (see Appendix A and Table III) are essentially the same as those observed in Daphnia.

\section{Possible Generalizations and Implications}

Growth efficiency of 20-28\% may be considered almost universal. Reproductive efficiency equal to slightly less than the square of the growth efficiency may also be very common. The maintenance cost per unit time $(y)$ will tend to vary with the amount of inert material present in the animal's body. For example, the larger the fat depot in the animals the lower will be the replacement rate, resulting in a correspondingly greater reduction in population size as a consequence of removing a single adult animal. The lower limit of production efficiency of adults is approximately $25 \%$. Replacement rate, and caloric content per animal, will vary from species to species. Actual growth efficiency probably will not vary very much. The energetic pattern of a population should then be largely determined from replacement rate, reproductive efficiency and the caloric content of the animals as a function of age.

\section{ConcluUsions}

It is possible to use steady state population data, caloric content data and an assumption of general linearity to determine growth efficiency, both including and not including maintenance cost, and reproductive efficiency. The numerical population equation was primarily significant in permitting us to assess approximate energetic input. In other situations it might be possible to assess energetic input into the population more directly in which case the analysis presented in the matrix (Appendix A) is not dependent on any numerical assumptions whatever and simply depends on caloric content as a function of age or size and on the conditions of the steady state population as far as size distribution and reproductive rate are concerned. The efficiencies determined from the Daphnia data are in surprisingly close agreement with biochemical values (Hutchens 1951) and, depending on the various interpretations that we give to the word efficiency, bracket all existing estimates of ecological efficiency (see Odum 1957 for examples). In essence, we have translated between ecological efficiency in the laboratory, biochemical efficiency determined in vitro, and field efficiency values. It would be expected that while replacement rate would vary from species to species the efficiency values for growth and reproduction which we have found will be approximately valid for all animals, or can be used as starting points until better analyses are available. The process of population maintenance in Daphnia is identical with that of replacing existing protoplasm with new protoplasm.

\section{AcKNowledgments}

The author would like to acknowledge the painstaking work of Miss Mimi Ho in making the actual population counts, the work of $\mathrm{Mr}$. Joseph Armstrong as Research Assistant, and the very valuable discussions with Mr. Joseph Armstrong, Drs. Frederick Smith, Karl Guthe, and Nelson Hairston of the Department of Zoology, University of Michigan, and Dr. Sumner Richman of Lawrence College. I would like to acknowledge Dr. Richman's kind permission to use his caloric content data prior to publication.

\section{Appendix}

The calculation of efficiencies, maintenance cost, and replacement rate from caloric content data.

A. Computation of production efficiencies and maintenance cost from an energy budget.

We define the following symbols: 


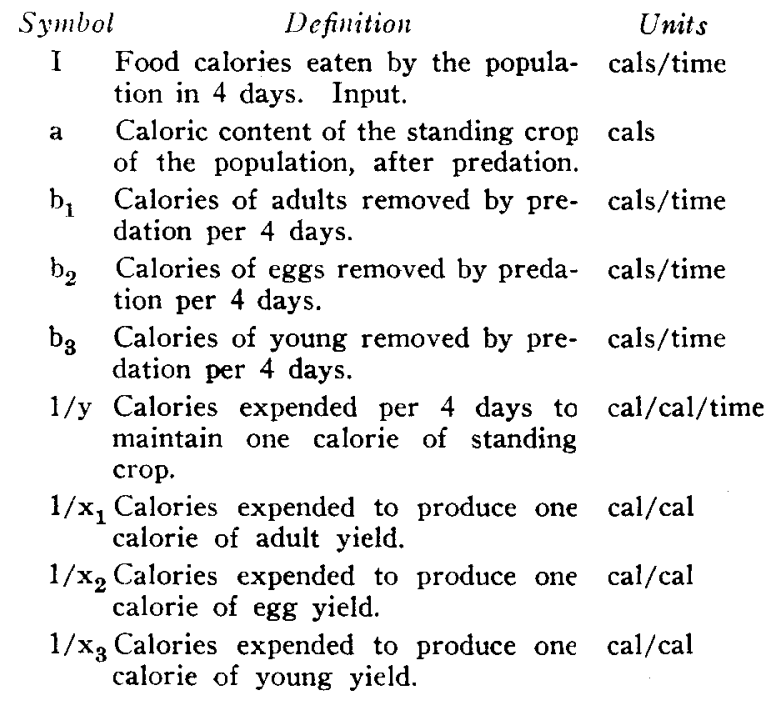

For any steady state population we can then write the equation:

$$
I=\frac{a}{y}+\left(\frac{b_{1}}{x_{1}}+\frac{b_{2}}{x_{2}}+\frac{b_{3}}{x_{3}}\right)
$$

Note that the symbols enclosed in parentheses in equation $3^{\prime}$ are equivalent to the words enclosed in parentheses in the verbal statement of the energy budget (equation (3) in the text (p. 238)). The constants $x_{1}, x_{2}$, and $x_{3}$ have the dimensions of efficiency and are the production efficiences of adults, eggs, and young respectively. That is, if a population did not have to expend energy for its own maintenance and was only producing adults for a predator to remove, it would produce adult tissue with caloric content equal to $x_{1}$ times the caloric input and similarly for the production of eggs and young.

We assume that $y$ and the $x_{i}$ 's are constant for all populations. The energy budgets of our 22 experimental populations can each be expressed in the form of equation ( 3 ) and these 22 equations can be reduced to a set of four linear equations :

$$
\begin{aligned}
& \sum_{1}^{22} a I=\sum a^{\mathrm{a}} \frac{1}{y}+\sum a b_{1} \frac{1}{x_{1}}+\sum a b_{2} \frac{1}{x_{2}}+\sum a b_{3} \frac{1}{x_{3}} \\
& \sum b_{1} I=\sum b_{1} a \frac{1}{y}+\sum b_{1}^{2} \frac{1}{x_{1}}+\sum b_{1} b_{2} \frac{1}{x_{2}}+\sum b_{1} b_{3} \frac{1}{x_{3}} \\
& \sum b_{2} I=\sum b_{2} a \frac{1}{y}+\sum b_{2} b_{1} \frac{1}{x_{1}}+\sum b_{2}^{2} \frac{1}{x_{2}}+\sum b_{2} b_{3} \frac{1}{x_{3}} \\
& \sum b_{3} I=\sum b_{3} a \frac{1}{y}+\sum b_{3} b_{1} \frac{1}{x_{1}}+\sum b_{3} b_{2} \frac{1}{x_{2}}+\sum b_{3}^{2} \frac{1}{x_{3}}
\end{aligned}
$$

which can be solved for the best least squares fit for $y$ and the $x_{i}$ 's. The solution has been done by constructing a $4 \times 4$ matrix in which each element $a_{i j}$ is equal to the $i, j^{\text {th }}$ coefficient of (4). This matrix was inverted by the Doolittle method and the inverse matrix $A^{-1}$ was then multiplied by the vector with elements

$$
g_{1}=\sum_{1}^{22} \frac{a I}{8.1} \quad, \quad g_{i>1}=\sum_{1}^{22} \frac{b_{i} I}{8.1}
$$

giving $8.1 \times \frac{1}{x_{i}}$ and $8.1 \times \frac{1}{y}$ directly as $\sum_{1}^{4} g_{i} a^{i, j}$.

$I$ was taken as predicted from equation (1) i.e., Column 8 of Table II. The matrix, its inverse, the vector and the solution for the $x_{\mathrm{i}}$ and $y$ are presented in Table III. The intermediate calculations have been omitted but the inversion procedure is clearly stated in Anderson and Bancroft (1952), pp. 191-197. Note that equation (3) is a special case of the generalized energy budget:

$$
I=\boldsymbol{\Sigma} M_{i} C_{m_{i}}+\boldsymbol{\Sigma} Y_{i} C_{y_{i}}
$$

where the $M_{i}$ 's are age, sex or genotype specific categorizations of the calories in the residual population and the $C m_{i}$ the corresponding costs for each category and the $Y_{i}$ 's are categories of yield calories with the $C y_{i}$ the corresponding costs. Equation (3) is used in its present form since analysis of a preliminary set of equations indicated that there was no difference in cost per calorie between the large and small animals in the residual population. The residual calories were therefore pooled and the control populations omitted from the analysis. When the values of $y$ and $x_{i}$ from the matrix solution are reinserted, for each population, in equation (3) and multiplied by the appropriate constants and summed a new estimate of $I$ is acquired. This new estimate of $I$ for each population is listed as Column 9 of Table II. If the matrix is re-solved, using the new estimate of $I$, no significant changes occur in our estimates of $y$ and the $x_{i}$.

B. Computation of replacement rate $(R)$ from efficiencies, maintenance cost, birth and standing crop data.

The rate at which the caloric content of the residual population is replaced can be computed as follows: 
We define $R$ as the replacement rate in calories per calorie per 4 days.

We define $\beta$ as the caloric content of the newborn produced per four days. This is computed for each population as the difference between the number born and the total number removed as yield multiplied by the caloric content of a newborn animal. Note that it could also be computed as the total number dead of non-predatory causes multiplied by the caloric content of one newborn.

The replacement calories occur as newborn animals $(\beta)$ and as new protoplasm added to the existing animals, presumably with an efficiency $x_{1}$.

The total energy available for replacement is $\frac{a}{y}$. The total energy available for growth is the difference between $\frac{a}{y}$ and the energy utilized for reproduction $\frac{3}{x_{3}}$. The calories of new protoplasm added by growth in four days are therefore

$$
x_{1}\left(\frac{a}{y}-\frac{\beta}{x_{3}}\right)
$$

and replacement rate for each population can then be computed as

$$
R=x_{1}\left(\frac{a}{y}-\frac{\beta}{\frac{x_{3}}{a}}\right)+\beta=\frac{x_{1}}{y}-\frac{\beta}{a}\left(\frac{x_{1}}{x_{3}}-1\right)
$$

All of the terms in equation (7) except for $\beta$ and $a$ are derived from the solution of equation (4) so that constancy of $R$ from population to population is a measure of the constancy of $\frac{\beta}{a}$. The values of $R$ for each population are listed as Column 11, Table II.

As a matter of fact, $R \approx y$, but this is an empirical agreement and is not analytically determinable from equation ( 7$)$.

\section{SUMmary}

Twenty-two populations of Daphnia pulex were subjected to predation in the laboratory. Five control populations were maintained. The steady state census and yield data and the food consumption of each population were translated into calories by use of conversion constants. Energy budgets were then written for each population by equating energy consumption to weighted caloric content values of the standing crop and yield of adults, eggs, and young. From the 22 energy budgets of the experimental populations, best least squares estimates were made for the weighting constants by a matrix inversion technique.

The weighting coefficients correspond to the respective inverses of the caloric replacement rate in the residual populations and the production efficiencies of adults, eggs, and young.

Efficiency in the various senses of ecological efficiency, production efficiency, and biochemical efficiency were then determined for the Daphnia populations. Each value was in agreement with correspondingly defined efficiency values in other systems. The implication of this agreement is that efficiency is approximately constant from species to species.

The replacement rate per calorie of standing crop was found to be identical with the maintenance cost per calorie of standing crop, implying that maintenance and replacement growth are identical processes.

\section{REFERENCES}

Anderson, R. L. and T. A. Bancroft. 1952. Statistical Theory in Research. New York: McGraw-Hill.

Armstrong, J. 1959. Doctoral dissertation (Zoology). The University of Michigan: Ann Arbor.

Beverton, R. J. H. and S. J. Holt. 1957. On the Dynamics of Exploited Fish Populations. Fishery investigations, Ministry of Agriculture, Fisheries and Food, Series 2, Volume 19, London.

Borecky, G. W. 1956. Population density of the limnetic Cladocera of Pymatuning Reservoir. Ecology 37: 719-727.

Hutchens, J. O. 1951. Machine efficiency of assimilative processes. Federation Proceedings 10: 622-628.

Juday, C. 1940. The annual energy budget of an inland lake. Ecology 21: 438-450.

Lindeman, $R$. 1942. The trophic-dynamic aspect of ecology. Ecology 23: 399-418.

Odum, H. T. 1957. Trophic structure and productivity of Silver Springs, Florida. Ecol. Monog. 27: 55-112.

Richman, S. 1958. The transformation of energy by Daphnia pulex. Ecol. Monog. 28: 273-291.

Slobodkin, L. B. 1954. Population dynamics in Daphitia obtusa Kurz. Ecol. Monog. 24: 69-88.

- - 1957. A laboratory study of the effect of removal of newborn animals from a population. Proc. Nat. Ac. Sci. 43: 780-782.

and S. Richman. 1956. The effect of removal of fixed percentages of the newborn on size and variability in populations of Daphnia pulicaria (Forbes). Limnology and Oceanography 1: 209-237.

Wright, J. C. 1958. The limnology of Canyon Ferry Reservoir. I. The phytoplankton-zooplankton relationships in the euphotic zone during September and October, 1956. Limnology and Oceanography 3: 150159. 